\title{
Supplemental Value of Topiramate in Infra Orbital Nerve Injury-Case Reports
}

\author{
Singh RK ${ }^{1 *}$, Pal US ${ }^{2}$, Sinha VP ${ }^{3}$ and Parveen Akther Lone ${ }^{4}$
}

${ }^{1}$ Professor, Department of Oral and Maxillofacial Surgery, KG Medical University Lucknow, India

${ }^{2}$ Associate Professor, Department of Oral and Maxillofacial Surgery, KG Medical University Lucknow, India

${ }^{3}$ Professor, Department of Oral and Maxillofacial Surgery, UP Dental College, Lucknow, India

${ }^{4}$ Assistant Professor, Department of Oral Surgery, Government Dental College Jammu, India

\begin{abstract}
This study was aimed to find out the supplemental value of topiramate therapy in infraorbital nerve paresthesia following post miniplate fixation of zygomatic complex fracture. Total four cases of unilateral zygomatic complex fracture which were one week old had infra orbital nerve paresthesia were treated. All the patients were treated with fracture reduction and miniplate fixation of zygomatic buttress region were supplemented with tab topiramate in therapeutic dosages. The dosages were increased depending upon tolerability and improvement. Functional recovery was observed postoperatively from first week and complete recovery was within three months of treatment.

Conclusion: We suggest that topiramate could be a useful supplemental form in the treatment of infra orbital nerve injury. Further studies (Double-blind) are needed with precise neurological examination to determine the recommendations of topiramate supplementation.
\end{abstract}

Keywords: ZMC fracture; Infraorbital nerve; Nerve injury; Sensory disturbance; Topiramate

\section{Introduction}

Zygomatico-orbital fractures are one of the most common facial injuries. Sakavicious et al. reported $64.4 \%$ incidence of infra orbital nerve injury in zygomatic-complex fracture [1]. Schilli reported that in $95 \%$ cases of ZC fractures, the fracture line involves the infra orbital foramen and may cause some degree of sensory disturbances [2]. Other studies [3] reported that the incidence of sensory disturbances in ZC fractures ranged from 30 to $80 \%$. The surgical management of infra orbital nerve injury requires decompression of nerve by reduction of zygomatic complex fracture and sometime mobilization of nerve surrounding soft tissue which helps in early recovery of sensory function. Smith-Swintosky et al. [4] claimed that topiramate helps in nerve regrowth and enhances the recovery of facial nerve function after injury when administered orally in therapeutically relevant doses, and significantly increases neurite outgrowth in cell cultures derived from fetal rat cortical and hippocampal tissues [5]. The purpose of this study was to find out the supplemental value of topiramate therapy in post miniplate fixation of zygomatic complex fracture.

\section{Literature Review}

Infra orbital nerve injury is mainly a compression type injury and results from zygomatic complex fracture with displacement at the infra orbital margin. This injury results compression of the nerve due to entrapments as it leaves the infra orbital foramen and in addition there is tissue swelling and nerve compression or traction also. Symptoms may vary from paresthesia to numbness at the site of the nose, upper lip, and heaviness or loss of sensation of affected site teeth. The presentation of paresthesia varies depending upon the severity of trauma and bony displacement. Several methods of sensory testing have been applied i.e. Gross mapping of altered areas of sensation is done. The conventional mechanical tests are Two-Point Discrimination (TPD), Light-Touch (LT), pinprick and sharp-blunt discrimination (SBD) [6]. Tests are carried out to assess their activity, however these tests are subjective and due to the variability in methodology and reporting, are of limited value. Results of these clinical (psychophysical) tests depend upon good communication between patient and doctor, and outcome assessment will depend on the patients' perceived experience and interpretation. Usually sensory disturbance examination of one site is sufficient. Examination of upper lip and its mucosa is an always accessible test site because functions like talking, eating, and drinking are affected when the upper lip is involved in a sensory disturbance. Zygomatic Complex Fracture Reduction And Fixation (ORIF) is a standard line of treatment to relieve symptoms. Few studies $[7,8]$ on the long term effects of treatment methods on sensory function have suggested that the treatment of isolated zygomatic complex fracture with open reduction and mini plate fixation, yields better recovery of sensory functions than other methods like open reduction and transosseous wiring or closed reduction without fixation. Benoliel et al. [9] reported use of topiramate in posttraumatic infraorbital neuropathic pain which responded favorably and sensory testing revealed a sign of nerve damage unchanged over the follow-up period parallel to an analgesic effect.

It has been observed that neurotropic agents like Vit B injection, methylcobalamin, gabapentin single or combination, topiramate and carbamazepine have been seen on patients' prescriptions. Various studies have reported the beneficial effects of Topiramate in posttraumatic nerve injury and in animal model topiramate promotes neurological recovery in rats after traumatic brain injury without affecting the final size of the traumatic lesion and that it might play a role in the reduction of post-traumatic cerebral edema $[4,5]$.

${ }^{*}$ Corresponding author: Singh RK, Professor, Department of Oral and Maxillofacial Surgery, KG Medical University Lucknow, India, E-mail: rksingh_9@rediffmail.com

Received November 23, 2012; Accepted January 17, 2013; Published January 19,2013

Citation: Singh RK, Pal US, Sinha VP, Lone PA (2013) Supplemental Value of Topiramate in Infra Orbital Nerve Injury-Case Reports. J Trauma Treat 2: 157. doi:10.4172/2167-1222.1000157

Copyright: ( 2013 Singh RK, et al. This is an open-access article distributed unde the terms of the Creative Commons Attribution License, which permits unrestricted use, distribution, and reproduction in any medium, provided the original author and source are credited. 
Topiramate is a compound with a broad spectrum of antiepileptic activities. It is proved clinically and experimentally. The main pharmacological action of this compound has been identified and is responsible for its anticonvulsant action by blockage of voltage-gated $\mathrm{Na}^{+}$channel, which reduces the duration and frequency of action potentials within spontaneous epileptiform bursts of neuronal firing Topiramate also has neuroprotective activity reported in rodents models of cerebral ischemia [1]. It also rescues oligdendrocytes in traumatic brain injury, stroke, epilepsy and periventricular leukomalacia. Topiramate can be prescribed in dosages of $50 \mathrm{mg}-400$ $\mathrm{mg}$ daily in divided dosages. Dosages can be increased by $25-50 \mathrm{mg} /$ day each subsequent week as tolerated. Based upon tolerability and improvement in paresthesia, additional titration to higher dose can be attempted in $25-50 \mathrm{mg} /$ day weekly increments.

Drug absorption is rapid, peak plasma concentration occurring at approximately two hours following a single $400 \mathrm{mg}$ oral dose. The plasma half life is $21 \mathrm{hrs}$ after single or multiple doses. TPM is not metabolized and primarily eliminates unchanged in the urine $(70 \%$ of the dose). The main side effects which require dose modification/ withdrawal are such as dizziness, blurred vision, Acute myopia and secondary angle glaucoma, oligohydrosis, metabolic acidosis, weight loss etc.

\section{Case Presentation}

In this study 04 case of zygomatic complex fracture with infraorbital nerve (ION) paresthesia were included, who were reported 5-7 days post injury. Chief complaints of the patients were reduced mouth opening and infra orbital nerve dysfunction. Detailed case history was taken. There was no significant medical history. All the patients did not had history of psychiatric illness. Physical examination revealed the step defect and tenderness present at the infra orbital margin and zygomatic buttress region. The mouth opening was less than $30 \mathrm{~mm}$ in all cases. Infra orbital nerve paresthesia was tested with two point discrimination test and cotton wick test. To test cold sensations, ethyl chloride vapour was sprayed onto a spherical dental cotton bud (diameter $5 \mathrm{~mm}$ ). After ice crystals were formed, the bud was placed on the test site for at least 1 second. Thermoreceptors and nocioceptive afferents may have been stimulated. On percussion of affected teeth there was heaviness/dullness. Conventional X-rays (Occipitomental and Submentovertex view of the skull) and the CT scan was carried out in some cases to confirm clinical diagnosis and to exclude other injuries. Routine blood investigations were also done, which were within the normal limit.

All the four patients were operated by consultant under general anesthesia. Gillies temporal approach and intraoral buccal incision was given to exposed fracture sites. The surgical decompression of the nerve was achieved by fracture reduction and single miniplate fixation was done at zygomatic buttress region. The mouth opening was achieved more than $40 \mathrm{~mm}$. The wound was closed in layers. Postoperatively antibiotic, supportive therapies were advised for seven days. The initial dose of Topiramate was $25 \mathrm{mg}$ /day administrated in night for the first week and then dose was increased to $50 \mathrm{mg} /$ day in the second week. Postoperatively patients were assessed clinically and radio logically. The patients were then recalled after $1^{\text {st }}$ week, $2^{\text {nd }}$ week, $4^{\text {th }}$ week, $8^{\text {th }}$ week and $12^{\text {th }}$ week for check up. On first week follow up, patients reported mild improvement in sensory function but teeth heaviness was present. Topiramate dose was increased to $50 \mathrm{mg}$ twice daily and further dose titration was advised to increase dosage by $25 \mathrm{mg}$ twice daily after one week depending upon tolerability and improvement and minimum dose was maintained. Minimum three months follow up was chosen in all patients, because most regenerative histologic reactions to trigeminal nerve damage is recovered within the above period. The patients were responding well on subsequent follow up with good recovery and remission of symptoms. After 12 weeks follow up, the patients reported complete recovery of nerve sensory function and absence of heaviness/pain during chewing. Sensory function related to temperature was considered normal if two successive positive responses in four tests were obtained.

\section{Discussion}

Zygomatic complex fracture commonly involves infra orbital nerve injury in majority of cases. Some patients often complained post fracture fixation sensory dysfunction. Such patients had to be reassured about their nerve recovery and were explained about the unpredictability of the nerve recovery period. In our study zygomatic fracture miniplate fixation was supplemented with Topiramate in therapeutic dosages for early nerve recovery as reported in the literature. Results were encouraging. Benoliel et al. [9] reported study of the 30 subjects in highly significant beneficial effect on nerve function, when plates were used to stabilize fractures relative to fractures that were reduced but not fixed. De Man and Bax [10] stated that reduction and fixation are important factors for the recovery of sensory disturbances in Infra orbital injury. Knight and North [7] also reported that fixation at FZ suture was found to be an optimal fixation point $[7,8,10]$. This method also caused indirect surgical decompression of the Infraorbital nerve and therefore obviates the need for direct decompression of the nerve. Above mentioned studies of the miniplate fixation at fronto zygomatic region have shown the evidence of nerve recovery, but in this study, fixation was done at zygomatic buttress region to avoid extra oral scar because fracture reduction was achieved by Gillis temporal approach and miniplate fixation was done intraorally. Patients were supplemented with Topiramate $25 \mathrm{mg}$ to $50 \mathrm{mg}$ twice daily, which helped in early recovery of sensory nerve function. Such type of study has not been reported in the literature so far. Many studies of either drug therapy or fracture miniplate fixation alone have been reported. Drug therapy also showed improvement in nerve function as reported Benoliel et al. [9] but takes longer period for complete recovery. This combination therapy showed an early improvement in infraorbital nerve function. This explains that a surgical exploration/reduction of fracture fragments prevents further damage to the nerve and supplemented drug (Topiramate) therapy helps in nerve regeneration as reported by many studies $[11,12]$.

\section{Conclusion}

From our study, we recommend an open reduction with miniplate fixation of zygomatic buttress region supplemented with Topiramate in therapeutic dosages enhances early recovery of sensory function of infra orbital nerve. A prospective double blind study with more precise neurological examination can give more authentic role of combination therapy.

\section{References}

1. Sakavicius D, Juodzbalys G, Kubilius R, Sabalys GP (2008) Investigation of infraorbital nerve injury following zygomaticomaxillary complex fractures. J Oral Rehabil 35: 903-916.

2. Schilli W (1990) Treatment of Zygomatic fractures. Oral Maxillofac Surg Clin North Am 2: 155-69.

3. Jungell $P$, Lindqvist $C$ (1987) Paraesthesia of the infraorbital nerve following fracture of the zygomatic complex. Int J Oral Maxillofac Surg 16: 363-367. 
Citation: Singh RK, Pal US, Sinha VP, Lone PA (2013) Supplemental Value of Topiramate in Infra Orbital Nerve Injury-Case Reports. J Trauma Treat 2: 157. doi:10.4172/2167-1222.1000157

Page 3 of 3

4. Smith-Swintosky, Virginia L. Zhao, Boyu Shank, Richard P, Plata-Salaman, et al. (2004) Topiramate promotes neurite outgrowth and recovery of function after nerve injury. J Peripher Nerv Syst 9: 70-8.

5. Kudin AP, Debska-Vielhaber G, Vielhaber S, Elger CE, Kunz WS (2004) The mechanism of neuroprotection by topiramate in an animal model of epilepsy. Epilepsia 45: 1478-1487.

6. Vriens JP, van der Glas HW, Bosman F, Koole R, Moos KF (1998) Information on infraorbital nerve damage from multitesting of sensory function. Int $\mathrm{J}$ Oral Maxillofac Surg 27: 20-26.

7. Knight JS, North JF (1961) The classification of malar fractures: an analysis of displacement as a guide to treatment. Br J Plast Surg 13: 325-339.

8. Taicher S, Ardekian L, Samet N, Shoshani Y, Kaffe I (1993) Recovery of the infraorbital nerve after zygomatic complex fractures: a preliminary study of different treatment methods. Int J Oral Maxillofac Surg 22: 339-341.

9. Benoliel R, Sharav Y, Eliav E (2007) Painful posttraumatic trigemina neuropathy: a case report of relief with topiramate. Cranio 25: 57-62.

10. De Man K, Bax WA (1988) The influence of the mode of treatment of zygomatic bone fractures on the healing process of the infraorbital nerve. $\mathrm{Br} \mathrm{J}$ Oral Maxillofac Surg 26: 419-425.

11. Fowler JA, Shen JY, Bettinger TL (2009) Successful use of topiramate in a patient with severe postherpetic neuralgia. Ann Pharmacother 43: 139-142.

12. Domingues RB, Kuster GW, Aquino CC (2007) Treatment of trigeminal neuralgia with low doses of topiramate. Arq Neuropsiquiatr 65: 792-794. 